\title{
GC-MS/MS analysis of metabolites derived from a single human blastocyst
}

\author{
Naomi Inoue ${ }^{1} \cdot$ Yoshihiro Nishida $^{1}\left[\right.$ [D $\cdot$ Emi Harada $^{1} \cdot$ Kumiko Sakai $^{1} \cdot$ Hisashi Narahara ${ }^{1}$
}

Received: 14 September 2020 / Accepted: 5 January 2021 / Published online: 25 January 2021

(c) The Author(s) 2021

\begin{abstract}
Introduction The field of assisted reproductive technology (ART) has significantly advanced; however, morphological evaluation remains as the chosen method of assessment of embryo quality.

Objective We aimed to examine metabolic changes in embryo culture medium to develop a non-invasive method for evaluation of embryo quality.

Methods We performed metabolic analysis of culture medium obtained from a single blastocyst cultured for freezing.

Results In total, 187 (39.8\%) of the 469 detectable organic acid metabolites were identified. A significant change $(p<0.05)$ was observed in eight metabolites between the good-quality and poor-quality embryo groups. Differences were observed in several metabolic pathways between the good-quality and poor-quality embryo groups. Metabolites that showed significant changes were primarily involved in the metabolism of branched-chain amino acids.

Conclusion The quantification of metabolism in human embryos may assist in identification and selection of good-quality embryos with high rates of survival before freezing and implantation in conjunction with morphological classification. This may help to identify embryos with high rates of survival.
\end{abstract}

Keywords Branched-chain amino acids $\cdot$ Culture medium $\cdot$ Embryo quality $\cdot$ Metabolomics

\section{Introduction}

Since the birth of the first child through in vitro fertilisation in 1978 (Steptoe and Edwards 1978), the field of assisted reproductive technology (ART) has considerably evolved, and outcomes for children born through this procedure continue to improve. In vitro fertilisation techniques have developed in multiple ways, including preimplantation diagnosis as a means of evaluating the embryo (Penzias et al. 2018). Currently, the morphological evaluation of embryos based on the classifications described by either Veek (1999) or Gardner and Schoolcraft (1999) is primarily used to assess embryo quality. However, the morphological evaluation of

Supplementary Information The online version of this article (https://doi.org/10.1007/s11306-021-01770-x) contains supplementary material, which is available to authorized users.

Yoshihiro Nishida

ynishida@oita-u.ac.jp

1 Department of Obstetrics and Gynecology, Faculty of Medicine, Oita University, 1-1 Idaigaoka, Hasama-machi, Yufu, Oita 879-5593, Japan embryos involves the subjective evaluation by each observer. Additionally, the morphology of the embryo does not necessarily reflect the successful implantation ability of the embryo. The number of studies examining non-invasive embryo evaluation methods, including the evaluation of genetic characteristics (Huang et al. 2019) and oxidation state (Alegre et al. 2019), has been increasing in recent years. To evaluate embryo quality, we analysed spent embryo culture medium for primary metabolites (organic acids, fatty acids and so on) absorbed or secreted by a single embryo. The purpose of this study was to perform exhaustive metabolomic analysis to subsequently analyse the differences in the metabolome between good- and poor-quality embryos and to evaluate the index of embryo development. Metabolic studies on embryos have been conducted using various animal species. One study providing a metabolic comparison of good- and poor-quality embryos in goats has been reported (Zhang et al. 2018); however, to the best of our knowledge, no study has simultaneously evaluated the morphological and exhaustive metabolic changes in a single human embryo using GC-MS/MS analysis. In this study, we aimed to examine the metabolic changes in culture medium 
obtained from a single blastocyst of each morphological classification, as determined by Gardner's classification, to add a quantitative evaluation of human embryo quality.

\section{Materials and methods}

\subsection{Collection of culture medium}

Embryo culture medium samples were collected from embryos produced by conventional in vitro fertilisation (cIVF), between January and December 2018 from the Oita University Department of Obstetrics and Gynecology, after normal fertilisation was confirmed. Embryos had been cultured for long-term freezing. In this study, samples were classified according to their morphological characteristics; the means of follicle stimulation were not included in the analysis. Informed consent was obtained from all individual participants (all couples who provided samples) by a unified document in this study. The couples' personal information was blinded, and samples were selected only for the morphological evaluation of the embryo. The study was approved by the Ethics Committee of Oita University Hospital (approval number 1317, Oita University).

\subsection{Sample preparation}

After confirmation of successful fertilisation (day 1), each embryo was cultured for 6 days (day 6 ) to form the blastocyst. Embryos were cultivated individually in a single continuous culture containing $70 \mu \mathrm{l}$ of broth medium. The samples were covered with oil to prevent evaporation of the nutrient solution. At the end of the culture, $50 \mu \mathrm{l}$ of embryo culture medium was collected from each embryo and stored at $-80{ }^{\circ} \mathrm{C}$.

The nutrient solution used was HiGROW OVIT (Fuso Pharmaceutical Industries Ltd.), with the addition of $10 \%$ Serum Substitute Supplement (Fuji Film Wako Pure Chemical Co., Ltd.). Embryos and cultures were added in 12- or 22-well plates (ART Culture dish, NIPRO) and cultured in a non-heating type dry incubator (EC65, ASTEC Inc.). Control samples included medium only and contained no embryos. Data on baseline characteristics of the female and male factors are shown in Supplementary 1.

\subsection{Morphological evaluation}

Morphological evaluation was performed by expert embryologists using an inverted microscope (ECLIPSE, TE300, Nikon Co., Japan). On day 6, we classified the embryos into three quality groups; good-quality embryos were those which scored a 3BB or above based on the Gardner classification, poor-quality embryos were defective embryos which scored lower than $3 \mathrm{BB}$, and embryos with developmental arrest were those with no visible growth (Table 1).

\subsection{Measurements}

Frozen embryo medium samples were thawed, phosphatebuffered saline was added to each $50 \mu$ culture sample to obtain a total volume of $200 \mu \mathrm{l}$, and lipids were extracted using the method reported by Bligh and Dyer (1959). An internal standard, $5 \mu$ of 2-isopropylmalic acid, was added, and centrifugation was performed for $7 \mathrm{~min}$ at $15,338 \times \mathrm{g}$. The organic acids in the top aqueous layer were recovered into a new tube and dehydrated to form pellets. Then, $40 \mu \mathrm{l}$ of methoxamine hydrochloride was added to facilitate the release of the pellets from the bottom of the tube by ultrasonic vibration. After shaking for $90 \mathrm{~min}$ at $30{ }^{\circ} \mathrm{C}, 20 \mu \mathrm{l}$ of $\mathrm{N}$-methyl-N-trimethylsilyl trifluoroacetamide (MSTFA) was added, and the mixture was subjected to shaking for a further $30 \mathrm{~min}$ at $37^{\circ} \mathrm{C}$. Following centrifugation, the supernatant was recovered and passed through a gas chromatographymass spectrometer (GC-MS/MS-TQ8040; SHIMADZU, Inc., Japan) to identify primary metabolites.

\subsection{Statistical analyse}

Statistical analysis was performed using multivariate analysis software (SIMCA13, Umetrics, Inc., Sweden). A $p$-value $<0.05$ indicated statistical significance. Orthogonal partial least squares regression discriminant analysis (OPLS-DA) was used to cluster embryo quality groups and controls, and S-plot analysis was used to compare significant changes in metabolites between quality groups. Metabolic set enrichment analysis (MSEA) and metabolic pathway

Table 1 Morphological data and day of classification of embryo samples used in this study

\begin{tabular}{cll}
\hline Group & Day of evaluation & Morphology \\
\hline Good & & \\
1 & Day 5 & $3 \mathrm{AB}$ \\
2 & Day 6 & $4 \mathrm{AB}$ \\
3 & Day 5 & $3 \mathrm{AA}$ \\
Poor & & \\
1 & Day 6 & $4 \mathrm{CC}$ \\
2 & Day 6 & $4 \mathrm{CC}$ \\
3 & Day 6 & $4 \mathrm{CC}$ \\
Death & & \\
1 & Day 6 & G4 3cell (arrest) \\
2 & Day 6 & G3 7cell (arrest) \\
3 & Day 6 & G4 3cell (arrest) \\
\hline
\end{tabular}

Based on the Gardner classification, 3BB is the threshold for goodquality embryos; below, $3 \mathrm{BB}$ is classified as poor-quality 
analysis (MetPA) were also used to confirm the significant pathways in the results of these measurements.

\section{Results}

Of the 469 organic acid metabolites that could be analysed by metabolic analysis, 187 (39.8\%) were identified. The metabolic profile of each embryo quality group, goodquality, poor-quality, developmental arrest (death), and control (medium only), was clearly identifiable by OPLSDA, which showed a clear distinction between the control and test groups (Fig. 1a, b). The distribution of good- and poor-quality groups was also statistically distinguishable (Fig. 1c). S-plot analysis of the good- and poor-quality groups (Fig. 1d) showed a difference in the level of metabolites between the two groups since the point was far from the center; 13 metabolites showed increased levels, while
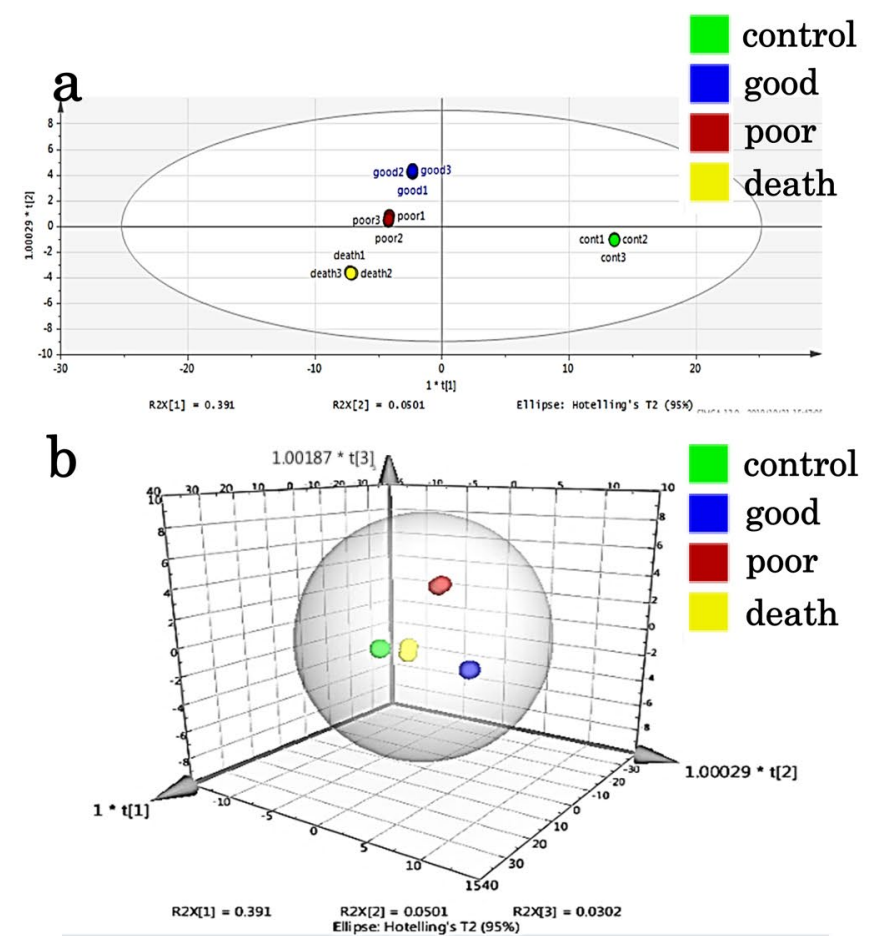

Fig. 1 Results of the multiple classification analysis using SIMCA13, Umetrics, Inc., Sweden. a Distribution map of the four quality groups by OPLS-DA. All samples of each group show similar distribution. The $\mathrm{x}$-axis $\mathrm{t}$ [1] and $\mathrm{y}$-axis $\mathrm{t}$ [2] represent score vectors summarising all variables of the analysis. The goodness of prediction value R2 [1] $=0.391$ and R2 [2] $=0.0501$. The ellipse shows the $95 \%$ confidence interval using Hotelling' T2 statistics. b 3D view of the four quality groups by OPLS-DA. The $\mathrm{x}$-axis $\mathrm{t}$ [1], y-axis $\mathrm{t}$ [2], and $\mathrm{z}$-axis $t$ [3] represent score vectors summarising all variables of the analysis. The goodness of prediction value $\mathrm{R} 2[1]=0.391, \mathrm{R} 2$ [2] $=0.0501$, and $\mathrm{R} 2[3]=0.0302$. The ellipse shows the $95 \%$ confidence interval
11 showed decreased levels in the poor-quality groups compared with good-quality groups. Additionally, six of the one hundred eighty metabolites were significantly increased $(p<0.05)$ while two were significantly reduced $(p<0.05)$ in the poor-quality group compared to those in the good-quality group (Table 2 ). The metabolic pathways identified as significant, using the Metabolite Set Enrichment Analysis (MSEA) and Metabolomic Pathway Analysis (MetPA), were involved in mitochondrial and amino-acid metabolism (Fig. 2a, b). Thus, we considered that the poor-quality group produced more energy than the good-quality group. Interestingly, both 2-hydroxyisovaleric acid and 2-hydroxyisocaproic acid levels were high in the poor-quality group and not included in culture media intrinsically. They are metabolites from valine, leucine, and isoleucine defined as branched-chain amino acids. Although valine, leucine, and isoleucine levels were lower in the poor-quality group, no significant difference was observed between the two groups (Fig. 3).
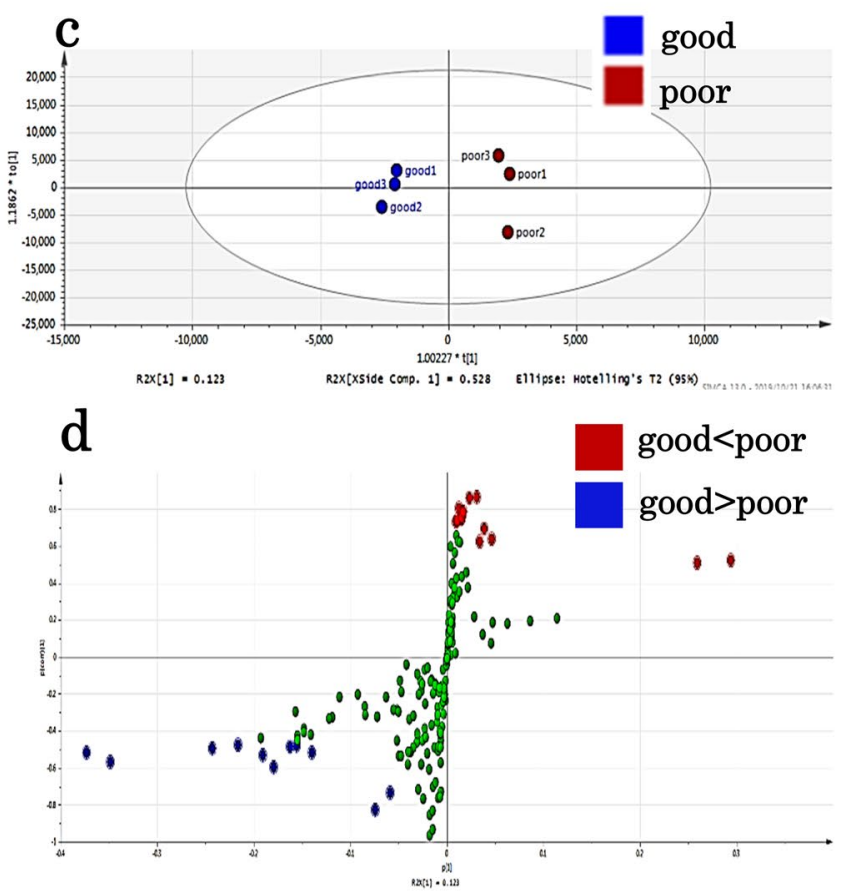

using Hotelling' T2 statistics. c Distribution map of the good-quality group vs poor-quality group by OPLS-DA. The distribution is remarkable between the good- and poor-quality groups. The $\mathrm{x}$-axis $\mathrm{t}$ [1] and $\mathrm{y}$-axis to [1] represent score vectors summarising all variables of the analysis. The goodness of prediction value $\mathrm{R} 2[1]=0.123$ and $\mathrm{R} 2$ to $[1]=0.528$. The ellipse shows the $95 \%$ confidence interval using Hotelling' T2 statistics. d S plot analysis of the good-quality group vs poor-quality group. OPLS-DA=orthogonal partial least square regression discriminant analysis. Positive or negative correlation levels are generally defined as $>0.8$ or $<-0.8$, respectively 
Table 2 Organic acids evaluated by S plot analysis

\begin{tabular}{|c|c|c|c|c|c|}
\hline \multirow[t]{2}{*}{ (a) } & \multicolumn{2}{|l|}{ Good } & \multicolumn{2}{|l|}{ Poor } & \multirow[t]{2}{*}{$p$ value } \\
\hline & Average & $\mathrm{SD}( \pm)$ & Average & $\mathrm{SD}( \pm)$ & \\
\hline 2-Hydroxyisovaleric acid & $10,208.67$ & 3265.685 & $21,972.67$ & 4317.334 & 0.018586 \\
\hline 3-Methylcrotonoylglycine & 3788.333 & 739.4964 & 5858.667 & 810.7183 & 0.027955 \\
\hline Dihydroxyacetone phosphate & 7125.667 & 1164.786 & $10,405.33$ & 1414.169 & 0.032277 \\
\hline Glycerol 3-phosphate & $19,412.33$ & 852.4828 & $22,957.67$ & 1831.116 & 0.034021 \\
\hline 5-Methoxytryptamine & 4254.333 & 311.1498 & 7999 & 2303.596 & 0.042477 \\
\hline 2-Hydroxyisocaproic acid & 9539.333 & 1782.132 & $13,382.33$ & 1780.663 & 0.048592 \\
\hline Pimelic acid & 3126.333 & 1064.42 & 4731.333 & 134.2543 & 0.050918 \\
\hline Citramalic acid & 3274 & 844.2859 & 4516.667 & 328.6846 & 0.062209 \\
\hline 3-Phenyllactic acid & $58,816.33$ & $16,009.84$ & $81,883.33$ & 7794.977 & 0.070451 \\
\hline Galactitol & 20,098 & 7013.909 & $58,162.33$ & $29,930.47$ & 0.077409 \\
\hline O-Phosphoethanolamine & $122,448.7$ & $10,774.51$ & 142,411 & $14,566.94$ & 0.097103 \\
\hline Dimethylglycine & 4079 & 1268.637 & 5470.667 & 499.4546 & 0.11118 \\
\hline 3-Hydroxyglutaric acid & $6,659,492$ & $1,660,659$ & $8,071,087$ & $568,138.8$ & 0.159442 \\
\hline \multirow[t]{2}{*}{ (b) } & \multicolumn{2}{|l|}{ Good } & \multicolumn{2}{|l|}{ Poor } & \multirow[t]{2}{*}{$p$ value } \\
\hline & Average & $\mathrm{SD}( \pm)$ & Average & $\mathrm{SD}( \pm)$ & \\
\hline Uridine & $154,391.3$ & $33,253.43$ & 80,985 & $10,369.91$ & 0.020363 \\
\hline Tryptamine & $156,819.3$ & $31,962.06$ & 104,001 & 8490.839 & 0.043403 \\
\hline Isoleucine & $11,970,899$ & $325,692.7$ & $11,351,353$ & $449,953.2$ & 0.094921 \\
\hline Hydroxylamine & $7,194,791$ & $443,351.5$ & $6,743,480$ & $180,671.4$ & 0.126671 \\
\hline Leucine & $28,682,819$ & $1,896,338$ & $26,448,798$ & $1,565,656$ & 0.134116 \\
\hline Glycine & $40,635,422$ & $601,859.1$ & $37,613,723$ & $3,416,155$ & 0.142719 \\
\hline Mannitol & $8,486,584$ & 609,098 & $7,751,260$ & $601,632.9$ & 0.145648 \\
\hline Allose & $7,892,075$ & $393,262.1$ & $7,305,359$ & $589,071.1$ & 0.153211 \\
\hline Mannose & $6,228,571$ & $342,985.6$ & $5,614,818$ & $668,722.7$ & 0.156211 \\
\hline Aspartic acid & $22,350,676$ & $501,649.8$ & $21,218,557$ & $1,305,195$ & 0.158028 \\
\hline Valine & $21,125,490$ & $1,643,932$ & $19,884,846$ & $287,107.8$ & 0.176205 \\
\hline
\end{tabular}

a Organic acid levels increased in the poor-quality group compared to those in the good-quality group. b Organic acid levels decreased in the poor-quality group compared to those in the good-quality group

\section{Discussion}

In this study, we comprehensively analysed metabolites of culture medium from a single blastocyst. Differences were observed in a few metabolic pathways in the poor-quality group compared with those in the good-quality group. We focused on amino-acid metabolism specifically. Amino acids are necessary for the development of embryos. The importance of amino acids in embryo culture medium has been reported by Brinstar in the mid-1960s (Brinster 1965). Thereafter, the requirement of amino acids for embryo development has been studied in various animal species including mice (Chatot et al. 1989; Ho et al. 1995; Nakazawa et al. 1997; Lane and Gardner 1998), rats (Miyoshi et al. 1995), sheep (Gardner et al. 1994), bovine (Rosenkrans et al. 1994; Lee and Fukui 1996), and pigs (Petters et al. 1990; Yoshioka et al. 2002). Several studies have reported that amino acids promote the differentiation of embryos in humans (Devreker et al. 2001; Winkle 2001; Houghton et al. 2002). Houghton et al. and Devreker et al. have reported that amino-acid metabolism is enhanced in embryos with arrested development compared to that in well-developing embryos. In the present study, it was observed that the morphological classification of embryos was correlated with amino-acid metabolism, and specifically that the metabolic pathways of valine, leucine, and isoleucine were enhanced in poorquality embryos. Our observation is consistent with that of previous studies reporting that metabolism is enhanced in poorly developing embryos. Metaboanalyst showed that valine, leucine, and isoleucine biosynthesis / degradation were concerned in differences of metabolites of poor- and good quality groups. Essential amino-acid metabolism as implied via valine, leucine, and isoleucine biosynthesis/ degradation was linked to the TCA cycle and was related to energy production (Supplementary 2). The metabolic pathways involved in mitochondrial metabolism were also 


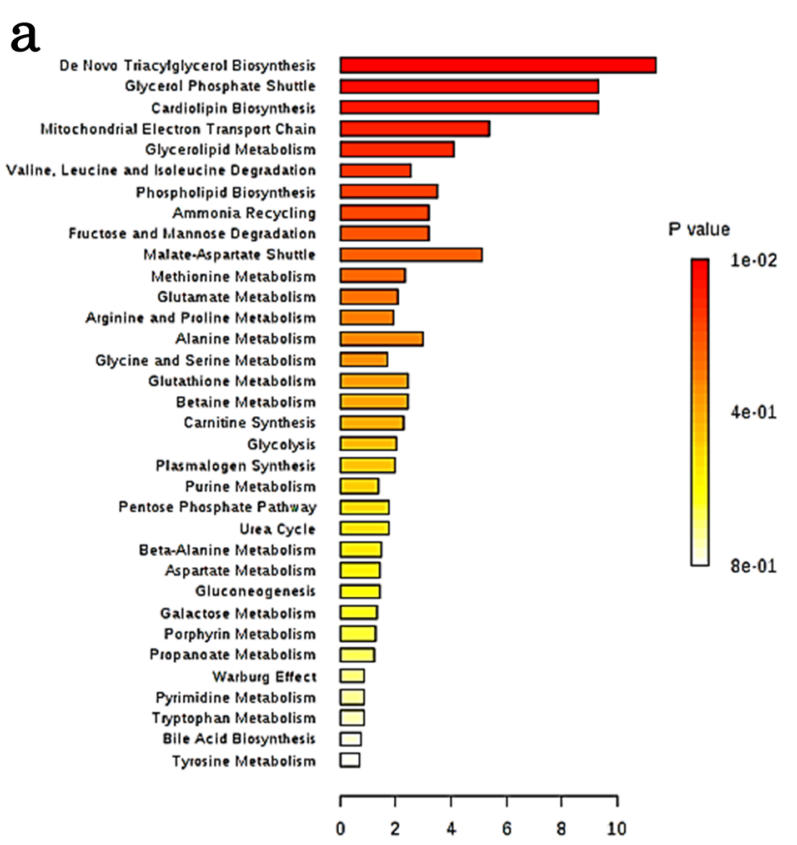

Fold enrichment

Fig. 2 Results of pathway analysis using MetaboAnalyst. a Result of metabolic set enrichment analysis (MSEA) showing threefold enrichment of the valine, leucine, and isoleucine degradation pathways. b Result of metabolic pathway analysis (MetPA). Pathway impact value (x-axis) from pathway topology analysis and $p$-values from b

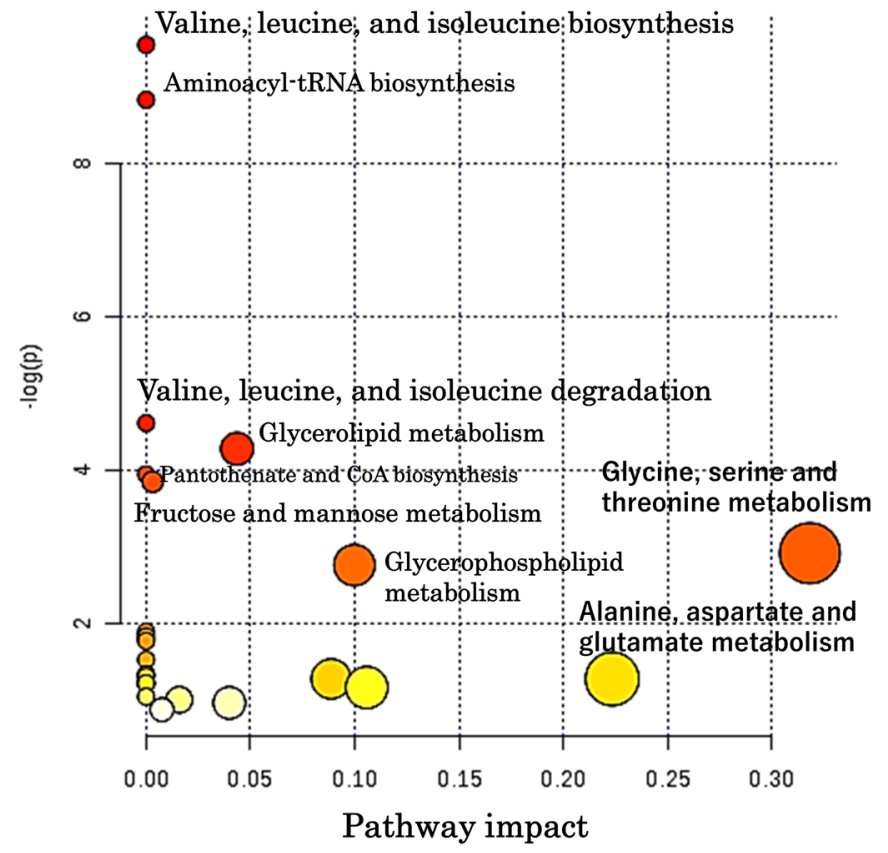

the pathway enrichment analysis (y-axis) are shown. MetaboAnalyst was developed by Dr. Jianguo Xia of the Institute of Parasitology at McGill University. It is a free software for metabolic analysis. (http:// www.metaboanalyst.ca/feces/home.xhtml)
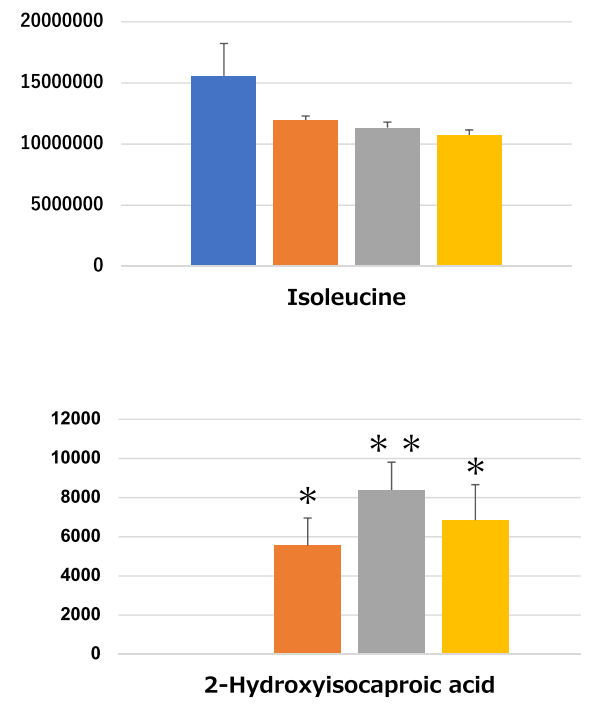

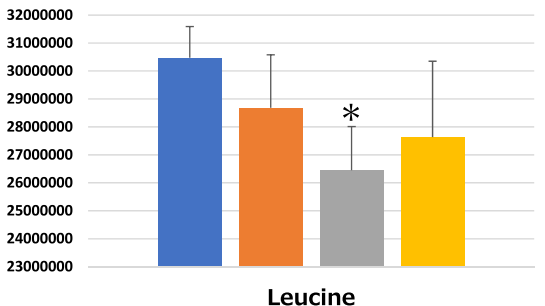

Leucine

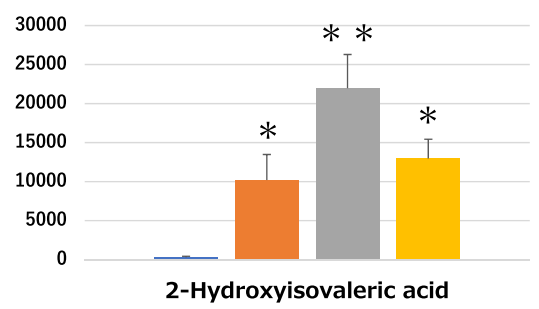

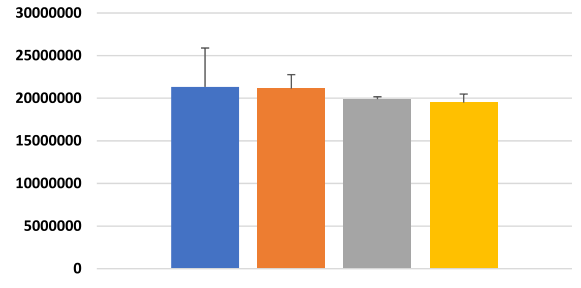

Valine
Fig. 3 Variation in metabolomic changes between the poor-quality and good-quality embryo groups. Valine, leucine, and isoleucine levels are lower in the poor-quality group compared with those in

enhanced in the poor-quality group. The poor-quality group requires more energy for the maintenance of cells than the good-quality group. the good-quality group. ${ }^{*} p$ value $<0.05$ compared with control. ${ }^{*} p$ value $<0.01$ compared with control

Bracewell-Milnes et al. (2017) have suggested a noninvasive embryo assessment to improve IVF outcome using spent culture medium, while others have suggested 
Raman, NMR, or NIR spectroscopy, and HPLC-MS. Uyar and Seli (2014) have indicated that GC-MS is a cumbersome procedure with long analysis time. Our GC-MS / MS technique required only a short period for analysis; therefore, there is a sufficient time to choose the best embryo suitable for transfer; hence we suggest that this method will be useful for assessment of the quality of embryos.

There were a few limitations of this study. The sample size was limited, and it was not possible to evaluate reproducibility using the same samples because all the available samples were used. In this study, the method of IVF was limited to cIVF, and different results might be obtained had the same study been carried out with ICSI. The study also did not examine pregnancy and labour; the establishment of pregnancy and the result of labour are determined not only by the morphological evaluation of the embryo but also by the involvement of various factors such as the intrauterine environment and maternal conditions. It is possible that a correlation between metabolic changes and pregnancy may be observed.

In this study, we analysed metabolites in the culture media derived from a single human embryo. Statistical analyses revealed that there were differences in metabolic pathways between poor- and good-quality embryos. This metabolome-based technique may thus be useful for the non-invasive and specific evaluation of embryo quality, in which changes in metabolites may be correlated with morphological classifications to select good-quality embryos. By performing this analysis prior to freezing the embryos, it may be possible to select embryos with a higher survival rate. However, it would be necessary to clarify the relationship between metabolites and pregnancy outcomes. We anticipate that the clinical application of our method may be used with morphological evaluation in the future.

Acknowledgements The authors would like to thank Dr. Yasushi Kawano (Oita University, Japan) for his valuable advice. This research was supported by JSPS KAKENHI [Grant Number JP19K22973 (YN), JP19K18646 (NI)] and the Smoking Research Foundation [Grant Number 2016G035 (YN)]. We would like to thank Editage (www.edita ge.com) for English language editing.

Author contributions NI, and YN designed this study, performed most of the experimental work, and drafted the article. EH collected the sample medium. KS performed metabolome analysis. HN proofread the manuscript.

Funding This research was supported by JSPS KAKENHI [Grant Number JP19K22973 (YN), JP19K18646 (NI)] and the Smoking Research Foundation [Grant Number 2016G035 (YN)].

Data availability The datasets generated during and/or analysed during the current study are available from the EMBL-EBI MetaboLights database (https://doi.org/10.1093/nar/gkz1019, PMID: 31691833) with the identifier MTBLS2189.

\section{Compliance with ethical standards}

Conflict of interest The authors of this study have no conflicts of interest to disclose.

Open Access This article is licensed under a Creative Commons Attribution 4.0 International License, which permits use, sharing, adaptation, distribution and reproduction in any medium or format, as long as you give appropriate credit to the original author(s) and the source, provide a link to the Creative Commons licence, and indicate if changes were made. The images or other third party material in this article are included in the article's Creative Commons licence, unless indicated otherwise in a credit line to the material. If material is not included in the article's Creative Commons licence and your intended use is not permitted by statutory regulation or exceeds the permitted use, you will need to obtain permission directly from the copyright holder. To view a copy of this licence, visit http://creativecommons.org/licenses/by/4.0/.

\section{References}

Alegre, L., Del Galego, R., Arrons, S., Hernández, P., Muñoz, M., \& Messenger, M. (2019). Novel noninvasive embryo selection algorithm combining time-lapse morph kinetics and oxidative status of the spent embryo culture medium. Fertility and Sterility, 111, 918-927.

Bligh, E. G., \& Dyer, W. J. (1959). A rapid method of total lipid extraction and purification. Canadian Journal of Biochemistry and Physiology, 37, 911-917.

Bracewell-Milnes, T., Saso, S., Abdalla, H., Nikolau, D., NormanTaylor, J., Johnson, M., et al. (2017). Metabolomics as a tool to identify biomarkers to predict and improve outcomes in reproductive medicine: A systematic review. Human Reproduction Update, 23, 723-736.

Brinstar, R. L. (1965). Studies on the development of mouse embryos in vitro. Journal of Reproduction and Fertility, 10, 227-240.

Chabot, C. L., Zoomed, C. A., Banister, B. D., Lewis, J. L., \& Torres, I. (1989). An improved culture medium supports development of random-bred 1-cell mouse embryos in vitro. Journal of Reproduction and Fertility, 86, 679-688.

Devreker, F., Hardy, K., Van den Bergh, M., Vanning, A. S., Emiliano, S., \& Angler, Y. (2001). Amino acids promote human blastocyst development in vitro. Human Reproduction, 16, 749-756.

Gardner, D. K., Lane, M., Spitzer, A., \& Batt, P. A. (1994). Enhanced rates of cleavage and development for sheep zygotes cultured to the blastocyst stage in vitro in the absence of serum and somatic cells: Amino acids, vitamins, and culturing embryos in groups stimulate development. Biology of Reproduction, 50, 390-400.

Gardner, D. K., \& Schoolcraft, W. B. (1999). In-vitro culture of human blastocysts. In R. Jansen (Ed.), Towards reproductive certainty: Fertility and genetics beyond (pp. 378-388). Cornforth: Parthenon Press.

Ho, Y., Wigglesworth, K., Epping, J. J., \& Schultz, R. M. (1995). Preimplantation development of mouse embryos in KSOM: Augmentation by amino acids and analysis of gene expression. Molecular Reproduction and Development, 41, 232-238.

Houghton, F. D., Hawkhead, J. A., McPherson, P. G., Hogg, J. E., Baleen, A. H., Rutherford, A. J., et al. (2002). Non-invasive amino acid turnover predicts human embryo developmental capacity. Human Reproduction, 17, 999-1005.

Huang, Lm., Bogle, B., Tang, Y., Lu, S., Xin, X. S., \& Racowsky, C. (2019). Noninvasive preimplantation genetic testing for aneuploidy in spent medium may be more reliable than trophectoderm 
biopsy. Proceedings of the National Academy of Sciences USA, 116, 14105-14112.

Lane, M., \& Gardner, D. K. (1998). Amino acids and vitamins prevent culture-induced metabolic perturbations and associated loss of viability of mouse blastocysts. Human Reproduction, 13, 991-997.

Lee, E. S., \& Fukui, Y. (1996). Synergistic effect of alanine and glycine on bovine embryos cultured in a chemically defined medium and amino acid uptake by in vitro-produced bovine morulae and blastocysts. Biology of Reproduction, 55, 1383-1389.

Miyoshi, K., Abeydeera, L. R., Okuda, K., \& Niwa, K. (1995). Effects of osmolality and amino acids in chemically defined medium on development of rat one-cell embryos. Journal of Reproduction and Fertility, 103, 27-32.

Nakazawa, T., Ohashi, K., Yamada, M., Shinoda, S., Saji, F., Murata, Y., et al. (1997). Effect of different concentration of amino acids in human serum and follicular fluid on the development of one-cell mouse embryos in vitro. Journal of Reproduction and Fertility, 111, 327-332.

Penzias, A., Bendikson, K., Butts, S., Coutifaris, C., Falcone, T., Fossum, G., et al. (2018). The use of preimplantation genetic testing for aneuploidy (PGT-A): A committee opinion. Fertility and Sterility, 109, 429-436.

Petters, R., Johnson, B. H., Reed, M. L., \& Archibong, A. E. (1990). Glucose, glutamine, and inorganic phosphate in early development of the pig embryo in vitro. Journal of Reproduction and Fertility, 89, 269-275.
Rosenkrans, C. F., Jr., \& First, N. L. (1994). Effect of free amino acids and vitamins on cleavage and developmental rate of bovine zygotes in vitro. Journal of Animal Science, 72, 434-437.

Steptoe, P. C., \& Edwards, R. G. (1978). Birth after the preimplantation of human an embryo. Lancet, 2, 366.

Uyar, A., \& Seli, E. (2014). Metabolomic assessment of embryo viability. Seminars in Reproductive Medicine, 32, 141-152.

Veek, L. (1999). An atlas of human gametes and conceptuses: An illustrated reference for assisted reproductive technology. Cleveland: CRC Press.

Winkle, L. J. V. (2001). Amino acid transport regulation and early embryo development. Biology of Reproduction, 64, 1-12.

Yoshioka, K., Suzuki, C., Tanaka, A., Anas, I. M., \& Iwamura, S. (2002). Birth of piglets derived from porcine zygotes cultured in a chemically defined medium. Biology of Reproduction, 66, $112-119$.

Zhang, Y. L., Zhang, G. M., Jia, R. X., Wan, Y. J., Yang, H., Sun, L. W., et al. (2018). Non-invasive assessment of culture media from goat cloned embryos associated with subjective morphology by gas chromatography-mass spectroscopy-based metabolomic analysis. Animal Science Journal, 89, 31-41.

Publisher's Note Springer Nature remains neutral with regard to jurisdictional claims in published maps and institutional affiliations. 